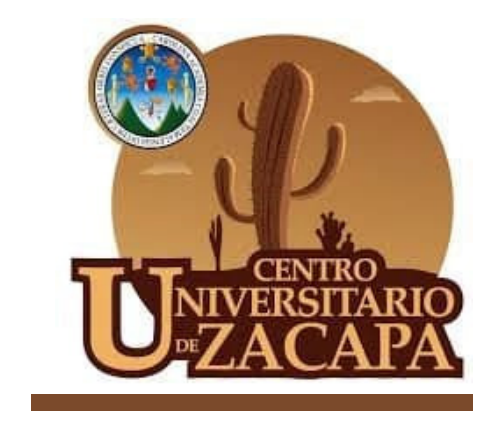

Fecha de presentación: 05/06/2020

Fecha de aceptación: 06/07/2020

Fecha de publicación: 30/11/2020

\title{
La innovación del aprendizaje en el contexto universitario
}

\author{
Joscelyne Priscila Arrieta Martínez \\ Universidad Rafael Landivar de Guatemala \\ priss5lc@gmail.com \\ https://orcid.org/0000-0002-1029-3543
}

Learning innovation in the university context

Referencia de artículo

Arieta, J. (2020). La innovación del aprendizaje en el contexto universitario. Revista Académica CUNZAC. 3(1) 53-56. DOI: https://doi.org/10.46780/cunzac.v3i1.19

\section{Resumen}

La innovación es la incorporación de novedades educativas a los procesos que se llevan a cabo, por la demanda de la sociedad que está en constante crecimiento, así mismo a las exigencias de las instituciones educativas universitarias. La reflexión que se realiza parte de la aplicación de la tecnología en el aula virtual, pero se propone explorar implicaciones que se encuentran en la base del fenómeno tecnológico basado en herramientas de internet que se utilizan en las universidades. El sistema educativo universitario, está indiscutiblemente inmerso en el fenómeno de la transformación tecnológica que se vive en la actualidad. Esta transformación ha originado drásticos cambios sociales y culturales. Entre ellos destacan los cambios en el campo de la comunicación, de la información y del conocimiento. Por lo tanto, el proceso educativo se ha visto influido por los múltiples cambios que representa la integración de las recientes tecnologías innovadoras de la virtual como lo expresa (Villela Cervantes, 2018). La integración de herramientas tecnológicas en las actividades didácticas es, sin lugar a dudas, ineludible y útil. Sin embargo, la integración didáctica de las diversas herramientas existentes, es el eslabón inmediato y visible del profundo impacto que la transformación tecnológica tiene en la educación universitaria.

Palabras clave: innovación, aprendizaje, contexto universitario

\begin{abstract}
Innovation is the incorporation of educational innovations into the processes that are carried out, due to the demand of society that is constantly growing, as well as the demands of university educational institutions. The reflection that is carried out starts from the application of technology in the virtual classroom, but it is proposed to explore implications that are at the base of the technological phenomenon based on internet tools that are used in universities. The university educational system is indisputably immersed in the phenomenon of technological transformation that is being experienced today. This transformation has brought about drastic social and cultural changes. Among them, the changes in the field of communication, information and knowledge stand out. Therefore, the educational process has been influenced by the multiple changes represented by the integration of the recent innovative technologies of the virtual as expressed (Villela Cervantes, 2018). The integration of technological tools in teaching activities is, without a doubt, unavoidable and useful. However, the didactic integration of the various existing tools is the immediate and visible link of the profound impact that technological transformation has on university education
\end{abstract}

Keywords: innovation, learning, university context 


\section{Introducción}

Los nuevos modelos centrados en las competencias, en las tutorías y los aprendizajes, debe partir de la práctica y utilización de la diversidad de herramientas que existen en internet, que en su mayoría son gratuitas, lo que se necesita es la actitud positiva del docente y de los estudiantes a través de aprendizaje autónomo. La innovación en la educación universitaria es la incorporación de novedades educativas a los procesos que se llevan a cabo, debido a la demanda de la sociedad que está en constante crecimiento e innovación, así mismo a las exigencias de las instituciones educativas superiores, que tiene como fin, la calidad educativa en la preparación de los profesionales en la diferentes áreas disciplinares.

En cuanto la reciente tecnología ha levantado un sinfín de barreras y límites. La manera de trabajar y aprovechar estas ventajas ha cambiado necesariamente el modelo de aprendizaje, anteriormente no se realizaban muchas videoconferencias para las clases virtuales, en la actualidad es lo que más se utiliza.

Las estrategias de aprendizaje también han cambiado significativamente para permitir diversas maneras de manipular y enfocar la información con herramientas de búsqueda, selección, recuperación y modificación de la misma. En cuanto al conocimiento se refiere, los cambios surgen del acceso a la información y el procesamiento de la misma. Es común hablar del desmedido incremento de información y de acceso a la misma. Hay que concentrarse más bien en las consecuencias de este hecho, un cambio en el concepto mismo de la adquisición, producción y transmisión del conocimiento. Las formas de aprendizaje están en un irrefrenable proceso de cambio ante el constante flujo de información $y$ ante las nuevas formas textuales de acceso a la información (Villela Cervantes, 2018).

\section{Materiales y métodos}

Se realizó investigación bibliográfica o documental citando autores de artículos científicos, se concluyó el proceso con la redacción a través de la reflexión hermenéutica.

\section{Resultados \\ Las innovaciones en educación}

Empezamos analizando el concepto de innovación según Botero (2008) es el conjunto de ideas, procesos y estrategias, sistematizadas, mediante los cuales se trata de introducir y provocar cambios en las prácticas educativas vigentes. La innovación no es una actividad puntual sino un proceso, un largo viaje o trayecto que se detiene a contemplar la vida en las aulas, la organización de los centros, la dinámica de la comunidad educativa y la cultura profesional del profesorado. Su propósito es alterar la realidad vigente, modificando concepciones y actitudes, alterando métodos e intervenciones y mejorando o transformando, según los casos, los procesos de enseñanza y aprendizaje. La innovación, por tanto, va asociada al cambio y tiene un componente, explícito, ideológico, cognitivo, ético y afectivo. Porque la innovación apela a la subjetividad del sujeto y al desarrollo de su individualidad, así como a las relaciones de las prácticas inherentes al acto educativo que tendrá un efecto en la relación del desempeño docente y el rendimiento académico de los estudiantes si se utiliza en forma efectiva la tecnología como lo asegura (Castañeda-Paz, M. I. y Villela-Cervantes, 2018).

Las mejoras que se obtienen con la innovación llevan varios objetivos; uno de ellos es optimizar el tiempo y los recursos para alcanzar las metas planteadas. Otro objetivo es unificar esfuerzos para conseguir un mismo fin. Asimismo, la incorporación de las TIC para facilitar el proceso educativo, tanto en

Volumen 3 | Número 1 |Enero - Diciembre 2020 
el aula virtual como fuera de ella. La calidad educativa es una de las mejoras constantes, ya que las nuevas demandas de la sociedad deben ser satisfechas con productos eficientes y eficaces de cara a la competitividad en el área de la educación.

Los cambios que pone en marcha la vertiginosa transformación tecnológica en la cultura y la sociedad son numerosas, variadas y vertiginosas como lo afirma (Villela Cervantes, 2018). En cuanto a la educación y al conocimiento se refiere, se puede partir de la implementación de nuevas formas de comunicación, estas conducen a nuevas necesidades y a nuevas maneras de interactuar. Al mismo tiempo, dichos cambios representan la adopción de nuevas formas de producir y transmitir el conocimiento. La forma de comunicarse ha cambiado a partir del uso de una amplia variedad de canales de transmisión. Estos crean nuevos entornos de comunicación dictados por las variadas modalidades de interacción tecnológica. Los entornos comunicativos conllevan un proceso de socialización y culturización con características propias y diversas de las tradicionales, lo cual cambia y define los hábitos comunicativos de las personas que los utilizan.

La integración de espacios interactivos en las plataformas virtuales como Moodle, Blackboard y otras que utilizan las universidades, para el aprendizaje colaborativo, por medio de habilitación de foros, fortalecen la competencia comunicativa de los estudiantes como lo afirma Prado (2001) y Méndez-Vásquez (2018), las nuevas formas de aprendizaje, se basan en la interacción y el trabajo cooperativo, fomentan la interdisciplinariedad y permiten un conocimiento global de la realidad, es decir, conforman un aprendizaje constructivo y significativo, y además facilitan el aprendizaje en general.
La innovación educativa para la universidad representa también crear nuevas carreras en ambientes virtuales de aprendizaje, de manera muy particular en el nivel superior, no pueden conformarse con proporcionar medios y acceso a la moderna tecnología al servicio del aprendizaje y del conocimiento. Es preciso asumir el contexto de estos cambios con el alto compromiso de adecuar la formación del estudiantado, para que de esta manera sean herramientas de desarrollo humano y evitar así su potencial nocividad según (Cañal, 2002).

\section{Discusión}

La innovación educativa permite introducir mejoras en las fases de los procesos de aprendizaje, se detecta la necesidad de cambio, con el objetivo de brindar un excelente servicio al estudiantado, en el menor tiempo posible y optimizando los recursos disponibles. La sociedad actual exige la innovación, en forma constante para renovar o acomodar procedimientos que anteriormente funcionaron, pero que ahora demanda agilidad, eficiencia y eficacia para satisfacer las solicitudes de autoridades, docentes, estudiantes y usuarios interesados en adquirir conocimientos de nivel superior de manera virtual. Sin embargo es fundamental que los docentes se capaciten en forma autónoma, teniendo acceso a Internet no debería existir motivo para autocapacitarse y también es importante que los docentes que vienen de la viejas prácticas, se actualicen en comprender y aceptar el respeto a la diversidad cultural, por que en lo complejo de la percepción del uso de la tecnología; los estudiantes, se encuentran los docentes con estudiantes que no les agrada encender la cámara al momento de las conferencias, porque aducen que violenta sus derechos individuales, de acuerdo a las costumbres arraigadas como lo describe Villela Cervantes, \& Gatica, (2018) es por ello

Volumen 3 | Número 1 | Enero - Diciembre 2020 
que como docentes se necesita comprensión en ese sentido, o saber utilizar las herramientas tecnológicas basadas en internet en forma efectiva, la comunicación expresiva que como competencia docente deben poseer los profesores, específicamente al momento que el docente necesita que los estudiantes enciendan la cámara, informar con anticipación para que se preparen y no tener inconvenientes con los estudiantes, poniendo como prioridad el respeto y comprensión y no como una orden que podría entenderse como abuso de autoridad.

\section{Referencias}

Castañeda-Paz, M. I. y Villela-Cervantes, C. E. (2018). Relación del desempeño docente y el rendimiento académico de los estudiantes. Revista Ciencia Multidisciplinaria CUNORI, 2(1), 41-46. https://doi. org/10.36314/cunori.v2i1.51

Cañal, P. (2002). La Innovación Educativa. Madrid: Akal.

Méndez-Vásquez, E. A. (2018). El tutor virtual y el aprendizaje colaborativo en ambientes tecnológicos. Revista Científica Internacional, 1(1), 43-46. https://doi. org/10.46734/revcientifica.v1i1.39

Prado, J. (2001). La competencia comunicativa en el entorno tecnológico: desafío para la enseñanza. Comunicar. 17, 21-30. Colectivo Andaluz para la Educación en Medios de Comunicación. Obtenido de http://www.redalyc.org/articulo. oa?id=15801704

Villela Cervantes, C. E. (2018). La educación virtual en las ciencias complejas. Revista Académica Cunzac, 1(1), 1-6. https:// doi.org/10.46780/cunzac.v1i1.1
Villela Cervantes, C. E., \& Gatica, S. (2018). Compilación de Bases Legales para la educación intercultural en Guatemala. Revista Académica Cunzac, 1(1), 15-38. https://doi.org/10.46780/cunzac.v1i1.3

\section{Sobre la autora}

Joscelyne Priscila Arrieta Martínez, es administradora de Empresas egresada de la Universidad Rafael Landivar de Guatemala, tiene un Máster en Docencia Universitaria, trabaja como docente en la maestría en Tecnología Educativa de la Facultad de Humanidades de la Universidad de San Carlos, en la Maestría en Administración Financiera del Centro Universitario de Oriente CUNORI de la Universidad de San Carlos, en la Facultad de Arquitectura en cursos de investigación en la Universidad Mariano Gálvez de Guatemala. Colabora desde hace 3 años en forma adhonorem como miembro del Comité de revisión de arbitraje de las siguientes revistas: Guatemalteca de Educación Superior, Revista Académica CUNZAC.

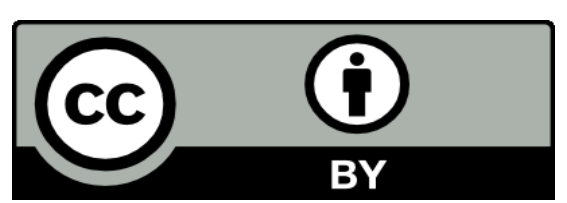

Este texto está protegido por una licencia Creative Commons 4.0.

Es libre para compartir, copiar y redistribuir el material en cualquier medio o formato y adaptar el documento, remezclar, transformar y crear a partir del material para cualquier propósito, incluso comercialmente, siempre que cumpla la condición de atribución: debe reconocer el crédito de una obra de manera adecuada, proporcionar un enlace a la licencia, e indicar si se han realizado cambios. Puede hacerlo en cualquier forma razonable, pero no de forma tal que sugiera que tiene el apoyo del licenciante o lo recibe por el uso que hace.

Volumen 3 | Número 1 Enero - Diciembre 2020 